\author{
Katarzyna Sztandarska \\ ORCID: 0000-0002-2046-6583 \\ Universität Szczecin, Szczecin
}

DOI: $10.19195 / 0435-5865.143 .25$

\title{
Phraseologismen aus dem Verdeutschungswörterbuch von Joachim Heinrich Campe (1801)
}

\begin{abstract}
s
Das Hauptanliegen des vorliegenden Beitrags richtet sich auf die Beschreibung von ausgewählten Phraseologismen, die aus dem Verdeutschungswörterbuch von Joachim Heinrich Campe (1801) stammen. Es werden Wortverbindungen aus dem Bereich der Äquivalente (Ersatzwörter/Ersatzausdrücke) dargestellt und interpretiert. Die Untersuchungen zeigen, dass manche Ausdrücke im Vergleich mit den festen Wortgruppen aus gegenwärtigen phraseologischen Wörterbüchern identisch verzeichnet sind; bei den anderen hat sich ihre Form und/oder Bedeutung verändert. Es werden auch solche Phraseologismen unterschieden, die im Gegenwartsdeutschen nicht mehr existieren.
\end{abstract}

Schlüsselwörter: Purismus in Deutschland, Verdeutschungswörterbücher, erklärend-verdeutschende Wörterbücher, Phraseologie

\section{Phraseologisms in the 'Germanizing dictionary' (Verdeutschungswörterbuch) by Joachim Heinrich Campe (1801)}

The aim of the paper is a description of selected phraseologisms taken from the 'Germanizing dictionary' (Verdeutschungswörterbuch) by Joachim Heinrich Campe (1801). Word combinations offered there as equivalents (words/expressions suggested as replacements) are presented and interpreted. The research shows that many expressions, when compared with fixed word groups from contemporary dictionaries, are recorded in the same form and with the same meaning; there are also some items whose form and/or meaning has changed. There are, moreover, such phraseologisms which do not exist in contemporary German any more.

Keywords: purism in Germany, 'Germanizing dictionaries', ‘explaining-Germanizing dictionaries', phraseology 
Katarzyna Sztandarska, Uniwersytet Szczeciński, Instytut Filologii Germańskiej, Al. Piastów 40b, bud. 5, pok. 103, 71-065 Szczecin, Polen, E-Mail: katarzyna.sztandarska@usz.edu.pl Received: 7.09.2017, accepted: 18.05.2018

\section{Einleitung und Zielsetzung}

Der Beitrag verfolgt das Ziel, ausgewählte Phraseologismen im Verdeutschungswörterbuch von Joachim Heinrich Campe zu untersuchen. Das Wörterbuch wurde im Jahre 1801 herausgegeben.

Campes Arbeit eröffnet eine Reihe von lexikographischen Werken, in denen Fremdwörter (fremde Ausdrücke) als Hauptlemmata durch heimischen Wortschatz oder völlig eingebürgerte Entlehnungen ersetzt werden (vgl. Lipczuk 2007: 45). Verdeutschungswörterbücher enthalten normalerweise keine phonetischen oder grammatischen Angaben. Zu dieser Gruppe gehören u.a. Werke von Karl Friedrich Dobel ([1825] 1833, 2. Aufl.), Hermann Dunger (1882), Daniel Sanders (1884), Otto Sarrazin ([1886] 1918, 5. Aufl.), Edward Lohmeyer ([1890] 1917, 3. Aufl.), Friedrich Düsel ([1915] 1915, 2. Aufl.), Oskar Kresse (1915) und Eduard Engel (1918) (vgl. Lipczuk/Sztandarska 2015a: 106).

$\mathrm{Zu}$ Wörterbüchern mit puristischen Zielen zählen auch Fremd- und Verdeutschungswörterbücher bzw. erklärend-verdeutschende Wörterbücher (vgl. Heier 2012). Hier werden fremdsprachige Wörter (oder Ausdrücke) sowohl erklärt als auch mit (meist) nativen Ersatzwörtern (Ersatzausdrücken) versehen, um den Gebrauch des Fremdwortes mindestens zu beschränken. Grammatische oder phonetische Informationen sind anzutreffen. Als erklärend-verdeutschende Wörterbücher gelten u.a. Werke von Johann Christian August Heyse ([1804] 1873, 15. Ausg.), Friedrich Erdmann Petri ([1806] 1929, 42. Aufl.), Johann Gottfried Sommer ([1814] 1819, 2. Ausg.), Günther A. Saalfeld (1898) und Albert Tesch (1915) (vgl. Lipczuk/Sztandarska 2015b: 183).

Zuerst werden im Artikel Phraseologismen aus dem Verdeutschungswörterbuch von Joachim Heinrich Campe (1801) dargestellt und interpretiert. Die Aufmerksamkeit richtet sich auf die festen Wortverbindungen aus dem Bereich der Äquivalente (Ersatzwörter/Ersatzausdrücke), weil diese besonders stark im Wörterbuch vertreten sind. Die Ausdrücke werden in drei Gruppen eingeteilt:

1) Phraseologismen mit keinen Veränderungen,

2) Wortverbindungen mit einigen Veränderungen in ihrer Form und/oder Bedeutung und

3) Phraseologismen, die heute in keinem zugänglichen phraseologischen Wörterbuch vorhanden sind.

Weiterhin wird im Falle der verdrängten Phraseologismen und Wortverbindungen mit Veränderungen geprüft, ob sie vielleicht in anderen Verdeutschungswörterbüchern oder erklärend-verdeutschenden Wörterbüchern in derselben Form 
und Bedeutung erscheinen. Untersucht werden folgende puristische lexikographische Arbeiten: Johann Gottfried Sommer (1819), Karl Friedrich Dobel (1833), Johann Christian August Heyse (1873), Hermann Dunger (1882), Daniel Sanders (1884), Günther A. Saalfeld (1898), Friedrich Düsel (1915), Oskar Kresse (1915), Albert Tesch (1915), Edward Lohmeyer (1917), Eduard Engel (1918), Otto Sarrazin (1918) und Friedrich Erdmann Petri (1929).

Im letzten Schritt werden Phraseologismen aus einigen ausgewählten Wörterbüchern mit puristischen Zielen besprochen. Unter diesen wird das Augenmerk gelenkt auf die festen Wortverbindungen, deren Form und Bedeutung beibehalten wurden und auf die Ausdrücke, bei denen zeitliche Veränderungen festgestellt werden können.

Bei Bedeutungs- und Strukturangaben halte ich mich an die Duden-Bände Redewendungen und sprichwörtliche Redensarten. Idiomatisches Wörterbuch der deutschen Sprache/Redewendungen. Wörterbuch der deutschen Idiomatik (Duden 11), Zitate und Aussprüche. Herkunft, Bedeutung und aktueller Gebrauch (Duden 12) und Das große Buch der Zitate und Redewendungen (Duden 2007). Außerdem benutze ich den Band Phraseologisches Wörterbuch Deutsch-Polnisch (Czochralski/Ludwig 2004) von Jan Czochralski und Klaus-Dieter Ludwig und Moderne deutsche Idiomatik. Alphabetisches Wörterbuch mit Definitionen und Beispielen (Friederich 1976) von Wolf Friederich. Die Bedeutungen der oft ausgestorbenen Fremdwörter prüfe ich in den Duden-Bänden Das Fremdwörterbuch. Unentbehrlich für das Verstehen und den Gebrauch fremder Wörter (Duden 5) und Das Herkunftswörterbuch. Etymologie der deutschen Sprache (Duden 7), bei Kluge Etymologisches Wörterbuch der deutschen Sprache (Kluge 2002) und in den gegenwärtigen Wörterbüchern der deutschen Sprache Duden. Deutsches Universalwörterbuch (Duden 2015) und Wahrig. Deutsches Wörterbuch (Wahrig 2011).

Den Gegenstand meiner Überlegungen bilden Phraseologismen im engeren Sinne, die drei Eigenschaften aufweisen: Polylexikalität, Festigkeit und Idiomatizität (vgl. Burger 2003: 11-32). Kollokationen und strukturelle sowie kommunikative Phraseologismen werden von mir nicht berücksichtigt. Ich vertrete die Ansicht, dass

ein Phraseologismus eine Wortverbindung ist, die mindestens ein autosemantisches Wort enthält; [...]. Deswegen entfallen Verbindungen der korrelativen Konjunktionen wie entweder oder und Präpositionen wie von - bis. Auch die feste Bindung eines Verknüpfungselements an ein Verb (Rektion), wie sie bei achten auf auftritt, konstituiert keinen Phraseologismus. (Sztandarska 2011: 282, in Anlehnung an Fleischer 1997: 29) 


\section{Phraseologismen aus dem Verdeutschungswörterbuch von Joachim Heinrich Campe (1801) ${ }^{1}$}

\subsection{Phraseologismen mit keinen Veränderungen}

Campes Verdeutschungswörterbuch registriert Phraseologismen im Bereich der Äquivalente (fett gedruckt), die heutzutage geläufig sind. Die angeführten Beispiele zeigen einerseits gelungene Wortverbindungen, die sich in der deutschen Sprache durchgesetzt haben. Andererseits kann man aber in diesem Fall von solchen Phraseologismen sprechen, die den Fremdwortschatz (in Klammern) nicht aus dem Gebrauch eliminiert haben und heute neben den Fremdwörtern (fremden Ausdrücken) als ihre Synonyme funktionieren, vgl.:

a) in die Augen fallen (figuriren)

heute: in die Augen fallen, Sinn: ,als Merkmal so offensichtlich sein, dass man es nicht übersehen kann, dass es sofort auffällt" (Duden 11, Czochralski/ Ludwig 2004, Friederich 1976) ${ }^{2}$

b) festen Fuß fassen (Posto fassen)

heute: festen Fuß fassen, Sinn: ,sich nach einer geraumen Zeit in einer neuen Umgebung integrieren [und durchsetzen]“" (Duden 11, Duden 2007, Czochralski/ Ludwig 2004, Friederich 1976)

c) Gleiches mit Gleichem vergelten (revanchiren)

heute: Gleiches mit Gleichem vergelten, Sinn: ,jmdm. dasselbe antun, was er einem zuvor angetan hat" (Duden 11, Duden 12, Duden 2007, Czochralski/ Ludwig 2004, Friederich 1976)

\subsection{Wortverbindungen mit einigen Veränderungen in ihrer Form und/oder Bedeutung}

Campes Verdeutschungswörterbuch enthält Phraseologismen als Ersatzausdrücke (fett gedruckt), die sich verändert haben. Die Veränderung ist bei ihrer Form und/ oder Bedeutung sichtbar. Beispiele:

a) sich etwas hinters Ohr schreiben (ad notam nehmen $)^{3}$

Campe ersetzt ad notam nehmen (Sinn: ,sich etwas merken“4) durch den Ausdruck sich etwas hinters Ohr schreiben. Die Bedeutung der - dem Autor zufolge -

\footnotetext{
${ }^{1}$ Alle Beispiele werden in originaler Schreibung vorgelegt. Wegen des Platzmangels können im Artikel nur ausgewählte Phraseologismen besprochen werden.

${ }^{2}$ In Klammern steht die Information, in welchen phraseologischen Wörterbüchern ein Phraseologismus gefunden wurde.

${ }^{3}$ Dasselbe Beispiel ist im Artikel von Sztandarska (2011: 285) zu finden.

${ }^{4}$ Vgl. Campe (1801: 131).
} 
einheimischen Wendung wird beibehalten. Die Form sieht aber heutzutage anders aus. Die Veränderung betrifft den Numerus. Die Wortverbindung lautet heute sich etwas hinter die Ohren schreiben (Duden 11, Duden 2007, Czochralski/Ludwig 2004, Friederich 1976).

Der von Campe vorgeschlagene Phraseologismus wird auch bei Autoren der anderen puristischen Wörterbücher registriert: Dunger (1882), Saalfeld (1898), Tesch (1915), Engel (1918) und Sarrazin (1918).

b) unter Einer Decke spielen (colludiren)

Das Fremdwort colludiren bedeutet bei Campe (1801: 236) „einverstanden sein". Das Verb kolludieren hat heute eine andere Bedeutung. Bei Wahrig (2011: 858) kann man lesen: ,zum Nachteil eines Dritten im Einverständnis mit jmdm. handeln".

Campe schlägt statt des Fremdwortes den Phraseologismus unter Einer Decke spielen vor, wo das Wort „Einer“ groß geschrieben ist. In den Duden-Bänden (Bd. 11, 2007), bei Czochralski/Ludwig (2004) und Friederich (1976) findet man den Ausdruck mit jmdm. unter einer Decke stecken (Sinn: ,,mit jemandem gemeinsame Sache machen, die gleichen [schlechten] Ziele verfolgen“, „,mit j-m gemeinsame Sache machen [in etw. Schlechtem]“, vgl. Duden 2007, Friederich 1976).

Im Falle des Phraseologismus lassen sich drei Unterschiede feststellen:

1. das Wort „einer" wird in den Duden-Bänden, bei Czochralski/Ludwig (2004) und Friederich (1976) klein geschrieben (bei Campe ist es Großschreibung),

2. eine andere lexikalische Besetzung liegt vor. Die Veränderung betrifft das Verb (Campe: „spielen“, gegenwärtige Wörterbücher: „stecken“),

3. die heutige Bedeutung des Ausdrucks ist breiter.

Anzumerken ist noch, dass Campes Phraseologismus in den puristischen Wörterbüchern der genannten Autoren nicht auftritt.

c) in die Queere kommen (contrecarriren)

Der im Wörterbuch gefundene Ausdruck in die Queere kommen (statt des Fremdwortes contrecarriren, Sinn: „entgegenarbeiten, hintertreiben“5) funktioniert heute in einer anderen Form. Die phraseologischen Wörterbücher (Duden 11, Duden 2007, Czochralski/Ludwig 2004, Friederich 1976) registrieren die Wortverbindung jmdm. in die Quere kommen, wo das Wort „Quere“ nur mit einem „E“ geschrieben wird. Der heute geläufige Ausdruck hat zwei Bedeutungen:

a) ,j-s Pläne, Arbeiten stören“,

b) ,jmdm. zufällig begegnen“" (Friederich 1976, Duden 11).

Die zweite Bedeutung war für Campe unbekannt.

Man kann hier auch feststellen, dass die geprüften puristischen Wörterbücher Campes Phraseologismus nicht angeben.

d) vom rechten Wege abführen (deroutiren)

${ }^{5}$ Vgl. Campe (1801: 261). 
Bei dem von Campe vorgeschlagenen Phraseologismus vom rechten Wege abführen - statt des Fremdwortes deroutiren, Sinn: ,irre leiten“6 - lässt sich ein lexikalischer Wandel feststellen. In den heutigen Wörterbüchern lautet die Wortverbindung jmdn. vom rechten Weg abbringen (Duden 11, Duden 2007, Friederich 1976).

In den untersuchten puristischen Wörterbüchern ist Campes Phraseologismus nicht anzutreffen.

e) zum Kreuze kriechen (submittiren)

Das Fremdwort submittiren bedeutet bei Campe (1801: 627),,sich unterwerfen“. Das Verb ersetzt der Autor durch die Wortverbindung zum Kreuze kriechen, die dieselbe Bedeutung, aber eine andere Form als die heute geläufige Wendung zu Kreuze kriechen (Duden 11, Duden 2007, Czochralski/Ludwig 2004, Friederich 1976) hat. Die Veränderung betrifft den Artikel. Nur in Campes Wörterbuch tritt der Phraseologismus mit der Präposition und dem bestimmten Artikel auf.

Festzustellen ist noch, dass der einheimische Ausdruck in den genannten puristischen Arbeiten nicht zu verzeichnen ist.

\subsection{Phraseologismen, die heute in keinem zugänglichen phraseologischen Wörterbuch vorhanden sind}

Campes Verdeutschungswörterbuch enthält solche Phraseologismen (im Bereich der Äquivalente), nach denen man vergeblich in gegenwärtigen phraseologischen Wörterbüchern sucht. Die unten angeführten Wortverbindungen sind in den Duden-Bänden (Duden 11, Duden 12, Duden 2007), bei Czochralski/Ludwig (2004) und Friederich (1976) nicht zu finden. Einige Beispiele:

a) das $A$ und $Z$ sein ( $A$ und $O$ sein)

Campe registriert den Phraseologismus $A$ und $O$ sein, der in den gegenwärtigen Wörterbüchern mit dem bestimmten Artikel auftritt. Die Wendung lautet das $A$ und $O$ und bedeutet ,die Hauptsache, das Wesentliche, der Kernpunkt, der Anfang und das Ende" (Duden 11, Duden 12, Duden 2007, Czochralski/Ludwig 2004, Friederich 1976). Der Autor war der Meinung, dass die sprichwörtliche Redensart $A$ und $O$ sein keineswegs passend ist, weil im Deutschen das „O" die Buchstabenreihe nicht schließt (vgl. Campe 1801: 117). Aus diesem Grunde sollte der Phraseologismus durch den einheimischen Ausdruck das A und $Z$ sein ersetzt werden.

Die von Campe vorgeschlagene Wortverbindung erscheint in keinem der zugänglichen heutigen Wörterbücher (Duden 11, Duden 12, Duden 2007, Czochralski/Ludwig 2004, Friederich 1976).

Der Phraseologismus ist auch in den geprüften lexikographischen Arbeiten mit puristischen Zielen unbekannt.

${ }^{6}$ Vgl. Campe (1801: 295). 


\section{b) zu Vieh machen (abrutiren)}

Der - nach Campe - einheimische Ausdruck zu Vieh machen ist in den heutigen phraseologischen Wörterbüchern (Duden 11, Duden 12, Duden 2007, Czochralski/Ludwig 2004, Friederich 1976) nicht zu finden.

Der Phraseologismus zum Vieh machen/werden (Sinn: „,[verthieren], verdummen") funktioniert nur in zwei erklärend-verdeutschenden Wörterbüchern, und zwar bei Heyse (1873) und Petri (1929).

c) zum stärksten Weingeist hinaufläutern (alcoholisiren)

Das Verb alkoholisieren bedeutet „mit Alkohol versetzen“ oder „,betrunken machen". ${ }^{7}$ Das Fremdwort wird durch die Wortverbindung zum stärksten Weingeist hinaufläutern nicht ersetzt. Der aus dem Wörterbuch von Campe stammende Ausdruck ist in keinem der zugänglichen heutigen phraseologischen Wörterbücher anzutreffen.

Die Wortverbindung zum stärksten Weingeist hinaufläutern (Sinn: ,vergeistigen“) findet man nur im Wörterbuch von Heyse (1873); bei Dobel (1833) und Petri (1929) die Form zum stärksten Weingeist läutern.

d) aus dem Groben arbeiten (ebauchiren)

Im Verdeutschungswörterbuch von Campe kommt das Fremdwort ebauchiren (Sinn: „leicht oder obenhin entwerfen“8) vor, statt dessen der Autor die Wortverbindung aus dem Groben arbeiten vorschlägt. Der einheimische Ausdruck erscheint aber nicht in der deutschen Sprache. Sowohl den Phraseologismus als auch das Fremdwort registrieren keine heutigen Wörterbücher der deutschen Sprache, keine phraseologischen und etymologischen Wörterbücher.

Der Ausdruck etwas aus dem Groben bearbeiten (Sinn: „leicht [od. obenhin] entwerfen, [vorzeichnen]") funktioniert im Verdeutschungswörterbuch von Dobel (1833) und im erklärend-verdeutschenden Wörterbuch von Heyse (1873). Dagegen verzeichnet Sanders (1884) die Wortgruppe aus dem Rohen (Groben) arbeiten (Sinn: ,entwerfen, die erste Hand anlegen").

e) den Tugendlehrer machen oder spielen (moralisiren)

Neben dem Fremdwort moralisiren (Sinn: ,predigen ${ }^{(9)}$ ) wird von Campe der Phraseologismus den Tugendlehrer machen oder spielen angegeben. Der Ausdruck hat sich im Gegenwartsdeutschen nicht durchgesetzt.

Anzumerken ist auch, dass die untersuchte Wortverbindung in derselben Bedeutung, aber in einer anderen (oft ähnlichen) Form in Verdeutschungswörterbüchern und erklärend-verdeutschenden Wörterbüchern auftritt. Vgl.:

Sommer (1819) und Dobel (1833) - den Sittenlehrer machen,

Dunger (1882) - den Tugendlehrer, Sittenlehrer spielen,

\footnotetext{
${ }^{7}$ Vgl. Duden (2015: 124).

${ }^{8}$ Vgl. Campe (1801: 318).

${ }^{9}$ Vgl. Campe (1801: 472).
} 
Sanders (1884) - den Sitten-Lehrer, -Meister, -Prediger, -Strafprediger, Rügemeister machen,

Saalfeld (1898) - den Sittenrichter spielen, den Tugendlehrer machen,

Düsel (1915) - den Sitten-, Tugendrichter spielen,

Tesch (1915) - den Sittenprediger spielen,

Lohmeyer (1917) - den Sittenrichter spielen,

Engel (1918) - sich zum (Sitten)Richter aufwerfen,

Sarrazin (1918) - den Sitten- od. Tugendlehrer od. -richter machen od. spielen.

Bei Heyse (1873: 598) wird der Phraseologismus vollkommen gleich dargestellt, vgl.: den Tugendlehrer machen od. spielen.

\section{Phraseologismen in anderen Wörterbüchern mit puristischen Zielen ${ }^{10}$}

Nicht nur im Verdeutschungswörterbuch von Campe, sondern auch in anderen Wörterbüchern mit puristischen Zielen werden Phraseologismen registriert.

Die untersuchten festen Ausdrücke im Bereich der Lemmata (fett gedruckt) sind oft lateinischer und französischer Herkunft. Sie stammen aus den Arbeiten von solchen Autoren wie Kresse (1915), Engel (1918) und Sarrazin (1918). Die Form und Bedeutung der gefundenen Wortverbindungen hat sich nicht verändert und sie werden in gegenwärtigen phraseologischen Wörterbüchern in gleicher Weise dargestellt und interpretiert, vgl.:

a) ab ovo (lat.)

heute: $a b$ ovo, Sinn: „sehr weitschweifig, von den allerersten Anfängen an“ (Duden 12, Duden 2007)

b) ad calendas graecas (lat.)

heute: ad calendas graecas, Sinn: „niemals“ (Duden 11, Duden 12, Duden 2007)

c) à la bonne heure (fr.)

heute: à la bonne heure, Sinn: „recht so, vortrefflich!“ (Duden 11)

d) alea iacta est (lat.)

heute: alea iacta est, Sinn: ,eine Entscheidung wurde getroffen [jetzt gibt es kein Zurück mehr]" (Duden 11, Duden 12, Duden 2007, Czochralski/Ludwig 2004, Friederich 1976)

In den puristischen Wörterbüchern (vgl. Kresse 1915, Lohmeyer 1917, Engel 1918 und Sarrazin 1918) funktionieren auch Phraseologismen als Äquivalente (Ersatzausdrücke), die in zwei Gruppen eingeteilt werden können. Es sind feste Wortverbindungen (fett gedruckt) zu finden, die in den heutigen phraseologischen

${ }^{10}$ Das Kapitel bezieht sich im großen Teil auf den Artikel von Sztandarska (2011: 281-288). Alle Beispiele stammen aus dem Artikel. 
Wörterbüchern in derselben Form und Bedeutung angegeben und verstanden werden. Einige Beispiele:

a) da lachen ja die Hühner (absurd)

heute: da lachen (ja) die Hühner, Sinn: „,das ist einfach lächerlich, unsinnig“ (Duden 11, Duden 2007, Friederich 1976)

b) in die Krone gefahren (ägriert)

heute: jmdm. in die Krone fahren, Sinn: ,jmdn. verärgern“ (Duden 11, Friederich 1976)

c) in den Schornstein geschrieben (à fonds perdu)

heute: etw. in den Schornstein/Schlot/in den/ins Kamin schreiben, Sinn: „etw. als verloren betrachten“ (Duden 11, Duden 2007, Czochralski/Ludwig 2004, Friederich 1976)

d) auf Gedeih und Verderb (à fonds perdu)

heute: auf Gedeih und Verderb, Sinn: „,bedingungslos, was auch Gutes oder Schlimmes geschehen mag“ (Duden 11, Duden 2007, Czochralski/Ludwig 2004, Friederich 1976)

e) sich ins Zeug legen (agitieren)

heute: sich ins Zeug legen, Sinn: „wer sich ins Zeug legt, strengt sich an“ (Duden 11, Duden 2007, Czochralski/Ludwig 2004, Friederich 1976)

f) Leben in die Bude bringen (animieren)

heute: Leben in die Bude bringen, Sinn: „für Unterhaltung, Spannung sorgen“ (Duden 11, Czochralski/Ludwig 2004, Friederich 1976)

g) schwarz auf weiß (authentisch)

heute: schwarz auf weiß, Sinn: ,gedruckt, schriftlich“ (Duden 11, Czochralski/Ludwig 2004, Friederich 1976)

Unter den Phraseologismen im Bereich der Ersatzwörter (Ersatzausdrücke) können auch solche Beispiele gefunden werden, bei denen sich Bedeutung (a) oder Form (b, c, d) verändert haben. Die festen Ausdrücke stammen aus dem Verdeutschungswörterbuch von Engel (1918). Beispiele:

a) nicht hintern Spiegel stecken (ad notam nehmen), Sinn: ,zur Kenntnis-, rot anstreichen, merken"

heute: sich etwas nicht hinter den Spiegel stecken, Sinn: „,beachten, zur Kenntnis nehmen“ (Duden 11), auch: ,durch eine scharfe Kritik o. Ä. in schriftlicher Form beschämt werden“" (Duden 11)

b) in Nummer Sicher bringen (arretieren)

heute: auf Nummer s/Sicher sein/sitzen, Sinn: ,im Gefängnis sitzen“(Duden 11, Friederich 1976)

c) in Fleisch und Blut assimilieren (assimilieren)

heute: jmdm. in Fleisch und Blut übergehen (Duden 11, Duden 12, Duden 2007, Czochralski/Ludwig 2004, Friederich 1976), Sinn: ,jmdm. zur selbstverständlichen Gewohnheit werden“ (Duden 11) 
d) unters alte Eisen werfen (ausrangieren)

heute: jmdn./etw. zum alten Eisen werfen/legen (Duden 11, Czochralski/Ludwig 2004, Friederich 1976), Sinn: ,jmdn., etw. als untauglich, als nicht mehr verwendungsfähig ausscheiden“ (Duden 11)

\section{Zusammenfassende Bemerkungen}

Das erste Wörterbuch, in dem versucht wurde, die Fremdwörter (fremde Ausdrücke) als Hauptlemmata durch heimischen Wortschatz oder völlig eingebürgerte Entlehnungen zu ersetzen, stammt von dem Lexikographen, Schriftsteller und Übersetzer Joachim Heinrich Campe. Das Verdeutschungswörterbuch wurde im Jahre 1801 herausgegeben (vgl. Lipczuk 2007: 45).

Die Untersuchungen zeigen, dass Campes Wörterbuch recht viele Phraseologismen im Bereich der Äquivalente (Ersatzwörter/Ersatzausdrücke) registriert. Bei manchen Wortverbindungen lassen sich keine Veränderungen feststellen (z.B. Gleiches mit Gleichem vergelten); bei den anderen wurde(n) ihre Form und/oder Bedeutung nicht beibehalten (vgl. unter Einer Decke spielen). Die puristische Arbeit registriert auch feste Wortgruppen, die in den gegenwärtigen phraseologischen Wörterbüchern nicht funktionieren (z.B. zu Vieh machen).

Es wurde festgestellt, dass manche von Campe vorgeschlagenen Wortverbindungen in anderen Verdeutschungswörterbüchern und erklärend-verdeutschenden Wörterbüchern erscheinen. In der Gruppe der Phraseologismen, bei denen Veränderungen in ihrer Form und/oder Bedeutung zu vermerken sind, findet man in den genannten puristischen Arbeiten nur einen Phraseologismus (sich etwas hinters Ohr schreiben). Dagegen ist für die Autoren der puristischen Wörterbücher die Mehrheit der - aus der heutigen Perspektive - ausgestorbenen festen Wortverbindungen in dergleichen oder ähnlichen Form nicht unbekannt (vgl. den Tugendlehrer machen od. spielen).

Im Beitrag wurden auch Phraseologismen aus anderen ausgewählten Wörterbüchern mit puristischen Zielen dargestellt und beschrieben. Die gefundenen Ausdrücke (im Bereich der Äquivalente) lassen sich ebenfalls in zwei Gruppen einteilen. Einerseits findet der Leser solche Phraseologismen, die heute identisch interpretiert werden (z.B. da lachen ja die Hühner); andererseits aber auch Ausdrücke, bei denen zeitliche Veränderungen zu beobachten sind (vgl. unters alte Eisen werfen). 


\section{Literatur}

\section{Quellen}

Campe, Joachim Heinrich (1801): Wörterbuch zur Erklärung und Verdeutschung der unserer Sprache aufgedrungenen fremden Ausdrücke. Ein Ergänzungsband zu Adelungs Wörterbuche. Braunschweig. (Reprint)

Czochralski, Jan / Ludwig, Klaus-Dieter (2004): Phraseologisches Wörterbuch Deutsch-Polnisch. Unter Mitarbeit von Undine Kramer. 2. Aufl. Warszawa.

Duden (1992): Redewendungen und sprichwörtliche Redensarten. Idiomatisches Wörterbuch der deutschen Sprache. Bd. 11, Mannheim etc.

Duden (2002): Redewendungen. Wörterbuch der deutschen Idiomatik. Bd. 11, 2. neu bearbeitete und aktualisierte Aufl. Mannheim etc.

Duden (2007): Das große Buch der Zitate und Redewendungen. 2. überarbeitete und aktualisierte Aufl., Mannheim etc.

Duden (2013): Redewendungen. Wörterbuch der deutschen Idiomatik. Bd. 11, 4. neu bearbeitete und aktualisierte Aufl. Berlin.

Duden (2017): Zitate und Aussprüche. Herkunft, Bedeutung und aktueller Gebrauch. Bd. 12, 4. überarbeitete und erweiterte Aufl. Berlin.

Friederich, Wolf (1976): Moderne deutsche Idiomatik. Alphabetisches Wörterbuch mit Definitionen und Beispielen. 2. Aufl., Ismaning.

\section{Sekundärliteratur}

Burger, Harald (2003): Phraseologie. Eine Einführung am Beispiel des Deutschen. 2. Aufl. Berlin.

Dobel, Karl Friedrich (1833): Verdeutschungsbuch der in unserer Sprache gangbaren fremden Wörter und Redensarten, nach der Buchstabenfolge geordnet. 2. Aufl. Kempten.

Duden (2007): Das Herkunftswörterbuch. Etymologie der deutschen Sprache. Bd. 7, 4. neu bearbeitete Aufl., Mannheim etc.

Duden (2010): Das Fremdwörterbuch. Unentbehrlich für das Verstehen und den Gebrauch fremder Wörter. Bd. 5, 10. aktualisierte Aufl. Mannheim etc.

Duden (2015): Deutsches Universalwörterbuch. 8. überarbeitete und erweiterte Aufl. Berlin.

Dunger, Hermann (1882): Wörterbuch von Verdeutschungen entbehrlicher Fremdwörter mit besonderer Berücksichtigung der von dem Großen Generalstabe, im Postwesen und in der Reichsgesetzgebung angenommenen Verdeutschungen. Mit einer einleitenden Abhandlung über Fremdwörter und Sprachreinigung. Leipzig.

Düsel, Friedrich (1915): Verdeutschungen. Wörterbuch fürs tägliche Leben. Zweiter unveränderter Abdruck, Braunschweig etc.

Engel, Eduard (1918): Entwelschung. Verdeutschungswörterbuch für Amt, Schule, Haus, Leben. Leipzig.

Fleischer, Wolfgang (1997): Phraseologie der deutschen Gegenwartssprache. 2. durchgesehene und ergänzte Aufl. Tübingen.

Heier, Anke (2012): Deutsche Fremdwortlexikografie zwischen 1800 und 2007. Zur metasprachlichen und lexikografischen Behandlung äußeren Lehnguts in Spachkontaktwörterbüchern des Deutschen. Berlin etc.

Heyses, Dr. Johann Christian August (1873): Allgemeines verdeutschendes und erklärendes Fremdwörterbuch mit Bezeichnung der Aussprache und Betonung der Wörter nebst genauer Angabe

Germanica Wratislaviensia 143, 2018

(C) for this edition by CNS 
ihrer Abstammung und Bildung. Neu bearbeitet, vielfach berichtigt und vermehrt von Prof. Gustav Heyse. 15. Ausg. Hannover.

Kluge, Norbert (2002): Etymologisches Wörterbuch der deutschen Sprache. 24. durchgesehene und erweiterte Aufl., Berlin etc.

Kresse, Oskar (1915): Verdeutschung entbehrlicher Fremdwörter mit Anhang: Deutsche Vornamen und ihre Bedeutung. Berlin.

Lipczuk, Ryszard (2007): Geschichte und Gegenwart des Fremdwortpurismus in Deutschland und Polen. Frankfurt am Main etc.

Lipczuk, Ryszard / Sztandarska, Katarzyna (2015a): Arabismen in deutschen Wörterbüchern. In: Colloquia Germanica Stetinensia 24. S. 103-121.

Lipczuk, Ryszard / Sztandarska, Katarzyna (2015b): Türkismen in deutschen Wörterbüchern. In: Mańczak-Wohlfeld, Elżbieta / Podolak, Barbara (Hrsg.): Words and Dictionaries. A Festschrift for Professor Stanisław Stachowski on the Occasion of His 85th Birthday. Kraków. S. 181-192.

Lohmeyer, Edward (1917): Unsere Umgangssprache. Verdeutschung der hauptsächlichsten im täglichen Leben und Verkehr gebrauchten Fremdwörter. 3. Aufl. Berlin. (Verdeutschungsbücher des Allgemeinen Deutschen Sprachvereins III).

Petri, Friedrich Erdmann (1929): Handbuch der Fremdwörter in der deutschen Schrift- und Umgangssprache unter Berücksichtigung der neuen Rechtschreibung, Erweiterung des Wortschatzes, Namendeuter, Erklärung der Wortkürzungen und Aussprachebezeichnung bearbeitet von Professor Dr. Rudolf Krauße. 42. Aufl. Leipzig.

Saalfeld, Günther A. (1898): Fremd- und Verdeutschungswörterbuch. Eine umfassende Sammlung von Fremdwörtern mit ausführlichen Verdeutschungen und sprachlichen Ableitungen nebst geschichtlicher Einleitung. Berlin.

Sanders, Daniel (1884): Verdeutschungswörterbuch. Leipzig.

Sarrazin, Otto (1918): Verdeutschungs-Wörterbuch. 5. vermehrte Aufl., Berlin.

Sommer, Johann Gottfried (1819): Neuestes wort- und sacherklärendes Verdeutschungswörterbuch aller jener aus fremden Sprachen entlehnten Wörter, Ausdrücke und Redensarten, welche die Teutschen bis jetzt, in Schriften und Büchern sowohl als in der Umgangssprache, noch immer für unentbehrlich und unersetztlich gehalten haben; verbunden mit einer Erklärung auch der weniger bekannten Kunstwörter und andern Ausdrücke der teuschen Sprache. Ein höchstnützliches Handbuch für Geschäftsmänner, Zeitungsleser, und alle gebildete Menschen überhaupt. Zweite durchaus umgearbeitete, verbesserte und sehr vermehrte Ausg., Prag. (Reprint)

Sztandarska, Katarzyna (2011): Phraseologismen im Verdeutschungswörterbuch von Eduard Engel. In: Lipczuk, Ryszard / Lisiecka-Czop, Magdalena / Misiek, Dorota (Hrsg.): Phraseologismen in deutsch-polnischen und polnisch-deutschen Wörterbüchern. Theoretische und praktische Aspekte der Phraseologie und Lexikographie (= Stettiner Beiträge zur Sprachwissenschaft 4). Hamburg. S. 281-288.

Tesch, Albert (Hrsg.) (1915): Fremdwort und Verdeutschung. Ein Wörterbuch für den täglichen Gebrauch. Leipzig etc.

Wahrig (2011): Deutsches Wörterbuch. Mit einem Lexikon der Sprachlehre. 9. vollständig neu bearbeitete und aktualisierte Aufl. Gütersloh etc.

Germanica Wratislaviensia 143, 2018

(C) for this edition by CNS 